\title{
Mundos Virtuais como Suporte à Aprendizagem - Uma Avaliação na Implementação de Projetos de Aprendizagem
}

\author{
Jadson do Prado Rafalski ${ }^{1,2,3}$ \\ Ramon Rosa Maia Vieira Júnior ${ }^{2,3}$ \\ Carlos Alexandre Siqueira da Silva ${ }^{2,3}$ \\ ${ }^{1}$ Faculdade Novo Milênio \\ Campus Vila Velha - Av. Santa Leopoldina ${ }^{\circ} 840$ - Coqueiral de Itaparica - Vila \\ Velha/ES - CEP 29102-040 \\ ${ }^{2}$ Departamento de Informática - Universidade Federal do Espírito Santo (UFES). \\ Av. Fernando Ferrari, 514, Goiabeiras - Vitória, ES, Brasil. CEP 29075-910. \\ ${ }^{3}$ Centro de Educação a Distância - Instituto Federal do Espírito Santo (IFES). Rodovia \\ ES-010, Km 6.5, Manguinhos, Serra, ES, Brasil. CEP 29173-087.
}

Resumo. Os mundos virtuais sempre foram utilizados nas áreas de jogos e ambientes de simulação, mas, atualmente, as pessoas estão vislumbrando outras possibilidades unindo o entretenimento e aprendizagem. Professores têm a possibilidade de utilizar os mundos virtuais como uma forma de tecnologia da informação e comunicação em sala de aula, para dinamizar as aulas apoiando o processo de aprendizagem, criando situações que levam o aluno à construção do conhecimento. Este trabalho apresenta uma contribuição ao uso de mundos virtuais para dar suporte à aprendizagem e é feita uma avaliação analisando o suporte tecnológico dos mundos virtuais para apoiar a realização de projetos de aprendizagem.

Palavras-chave: Mundos Virtuais. Projeto de Aprendizagem. Aprendizagem.

Abstract. Virtual worlds have always been used in the areas of gaming and simulation environments, but now people are envisioning other possibilities combining entertainment and learning. Teachers have the option of using virtual worlds as a form of information technology and communication in the classroom, to boost classes supporting the learning process, creating situations that leads the student knowledge building. This work presents a contribution from the use of virtual worlds to support learning and an assessment is made analyzing the technological support of virtual worlds to support the achievement of learning projects.

Keywords: Virtual Worlds. Learning Project. Learning.

\section{Introdução}

Os mundos virtuais (MVs) são bastante utilizados hoje nas áreas de jogos digitais e simuladores, e são capazes de apoiar as práticas de ensino-aprendizagem, propiciando interações com seus elementos, e a tomada de decisões, que serão avaliadas segundo os axiomas ali existentes. Atualmente, as pessoas têm vislumbrado outras possibilidades, como socialização, educação e entretenimento.

Segundo (Daden, 2010), entende-se por MV:

“[...] um ambiente digital, disponível na Internet ou Intranet, onde usuários: são representados por avatares; podem interagir entre si; podem interagir e afetar o ambiente 
no qual estão, de maneira persistente; Não têm restrições maiores impostas aos usuários além das existentes no mundo real; podem escolher entre um grande conjunto de ações ou inações; podem construir e criar no mundo, sem dominar ferramentas adicionais; podem utilizar o mundo segundo uma grande variedade de propósitos”. (DADEN, 2010).

Embora se tenham indícios de que os MVs possam ser ferramentas capazes de aprimorar o processo de aprendizagem (Prensky, 2001), e que este tipo de recurso começa a atrair a atenção de professores e alunos, é possível que não se conheça o grau de contribuição que os jogos e MVs podem trazer para a construção do conhecimento.

Neste trabalho utiliza o uso dos mundos virtuais para facilitar e dar suporte à promoção da aprendizagem realizando uma avaliação na implementação de Projetos de Aprendizagem. Verificaremos se é possível, nos MVs, os professores criarem/desenvolverem situações de aprendizagem que levem os estudantes a exercerem autoria e cooperação.

O artigo está dividido da seguinte maneira: o capítulo 1 corresponde a esta introdução, seguido pelo capítulo 2 que abordará aprendizagem com os mundos virtuais. O capítulo 3 traz os requisitos do mundo virtual. No capítulo 4 identificamos o conceito de projeto de aprendizagem. O capítulo 5 traz os mundos virtuais avaliados e elicitamos os requisitos para avaliação. O capítulo 6 avaliamos e mostramos os resultados. O capítulo 7 traz nossas conclusões, seguidas das referências utilizadas.

\section{Promovendo Aprendizagem com os Mundos Virtuais}

Os Mundos Virtuais podem fornecer novos horizontes para a construção de projeto de aprendizagem (Schlemmer e Backes, 2007), uma vez que, nos mundos virtuais, o estudante é um agente ativo, que constrói seu conhecimento na interação entre sujeito e objeto.

Desta maneira, não há momento nem lugar para que a aprendizagem aconteça, o que simula, de fato, o mundo físico, por meio da experimentação. Estudantes deixam de ser meros espectadores dos acontecimentos, outrora exclusivos à sala de aula, para serem protagonistas do processo de aprendizagem em ambientes de autoria e cooperação. Os mundos virtuais são novas formas de utilizar na integração com a aprendizagem, em que os estudantes têm a possibilidade de se relacionar, trocando informações e experiências (Gee, 2003).

Uma das possibilidades educacionais que estão em discussão atualmente é a forma de aprender utilizando os jogos digitais. Uma vez que o aprendizado pode se tornar mais divertido, torna-se, por sua vez, uma ferramenta motivacional com a qual as pessoas têm a possibilidade de aprender brincando. O Digital Game-Based Learning (DGBL) é a união de conteúdos de aprendizagem com jogos motivadores (Prensky, 2007).

\footnotetext{
“[...] O aprendizado baseado em jogos digitais (Digital Game Based Learning DGBL) está baseado em duas premissas: os aprendizes mudaram em diversos pontos fundamentais $e$ são de uma geração que experienciou profundamente, enquanto crescia, pela primeira vez na história, uma forma radicalmente nova de jogar computadores e videogames. Assistimos então a uma descontinuidade, inclusive na maneira como essas gerações aprendem. Por isso, boa parte dos dados que colhemos e das teorias que formulamos no passado, sobre como as pessoas pensam e aprendem, podem não se aplicar mais. Devemos levar em consideração novos estilos de aprendizagem. DGBL, ainda utilizado timidamente, não é o único método, mas é um método que consegue atingir essa nova geração. E o DGBL não serve apenas para atividades de revisão, mas para o aprendizado efetivo de diversos temas.” (MATTAR, 2009)
} 
Gee (2007) afirma que jogos digitais podem desenvolver habilidades cognitivas de seus jogadores e que esses são capazes de transferir o conhecimento adquirido do mundo virtual para o mundo real. Logo, com a devida abordagem pedagógica, GBL se mostra uma solução plausível para os problemas de desinteresse e falta de entusiasmo dos estudantes para estudar algumas áreas do conhecimento.

\section{Requisitos do Mundo Virtual}

Conforme Bell (2008), um MV é uma rede persistente, representado por avatares e facilitado pela tecnologia. Esta combina 5 cinco elementos principais que são discutidos por Bell (2008) baseado no conceito de um MV, que são: Sincronismo, Persistência, Rede de Pessoas, Representação por Avatares e Suporte Tecnológico.

Sincronismo parte das atividades compartilhadas e permite a ação de um grupo de massa e outras atividades sociais coordenadas. Os mundos virtuais oferecem, também, uma consciência de distância, espaço e coexistência de outros participantes encontrados em espaços da vida real.

Persistência é a ideia do MV não ser interrompido, ele continua a existir e funcionar após o participante deixá-lo, diferente dos jogos nos quais o mundo termina quando o jogador sai do jogo. Nesse mundo, o participante não é mais um mero espectador do centro do mundo, mas um membro de uma comunidade dinâmica. Os MVs podem existir com ou sem a presença de um participante. Todos os conteúdos de um mundo virtual devem ser preservados entre as sessões dos usuários e também quando o sistema é desativado momentaneamente para manutenção.

Rede de Pessoas é fundamental para os MVs, os participantes comunicam e interagem uns com os outros e com o meio (mundo), e podem formar grupos sociais.

Representação por Avatares é qualquer representação digital gráfica ou textual e que tem capacidade de realizar ações no mundo, e é controlado por um humano em tempo real.

Suporte Tecnológico é importante para que o mundo possa ser gerado de forma síncrona, persistente e com a representação por avatares. Além disso, o computador mantém o registro de todas as conversas, conexões e redes sociais de pessoas, permitindo-lhes a comunicação instantânea independente do espaço localizado (Bell, 2008).

Mundos Virtuais têm por finalidade, em sua concepção, oferecer um suporte tecnológico para apoiar as práticas de ensino-aprendizagem provendo, entre outros, recursos para facilitar a interação dos participantes. Entretanto, muitos desses ambientes, conforme Pessoa e Menezes (2003) requerem adaptação das práticas pedagógicas quando implementadas aos MVs, ou, ainda, um aumento do esforço cognitivo na apropriação de novas ferramentas para suprir as necessidades de um grupo de aprendizes quando estes ambientes não apoiam suas necessidades de trabalho.

\section{Projetos de Aprendizagem}

Projetos de aprendizagem (PAs), propostos por Fagundes et al. (1999), trata-se de uma metodologia em que o estudante deixa de ser mero expectador dos acontecimentos outrora exclusivos à sala de aula, para ser protagonista do processo de aprendizagem, por meio de ambientes de autoria e cooperação. Nesta abordagem, o estudante tem a oportunidade de construir conhecimento a partir da exploração de uma questão de 
investigação.

Segundo Fagundes et. al. (2006), é afirmado que projeto de aprendizagem tem por objetivo a:

[...]busca por informações que esclareçam as indagações de um sujeito sobre a sua realidade. Essas indagações se manifestam por inquietações advindas de suas vivências $e$ necessidades em conhecer e explicar o mundo. O objetivo é o desenvolvimento de um processo de aprendizagem que alcance a construção de novos conhecimentos, em que o aprendiz possa sistematizar informações ampliando sua rede de significações, possa reestruturar o raciocínio lógico sobre novos significados enquanto elabora síntese de respostas descritivas e explicativas para sua curiosidade.” (Fagundes, 2006).

Conforme apresentado por Carvalho et al. (2005), as seguintes etapas estão na figura 1: a) o professor em conjunto com seus alunos decide que temas gostariam de estudar, b) a partir dos temas escolhidos, os alunos reúnem-se em grupos e levantam questões de investigação - suas dúvidas temporárias, também levantam suas certezas provisórias, c) os alunos devem negociar, sendo que, muitas vezes, as certezas tornam-se dúvidas, d) início da pesquisa, e) o professor faz questionamentos e acompanha o desenvolvimento do projeto, f) relatório de pesquisa enfocando suas dúvidas, g) os visitantes têm a oportunidade de ver os projetos, participar de fóruns e responder a enquetes, e h) publicação dos projetos.

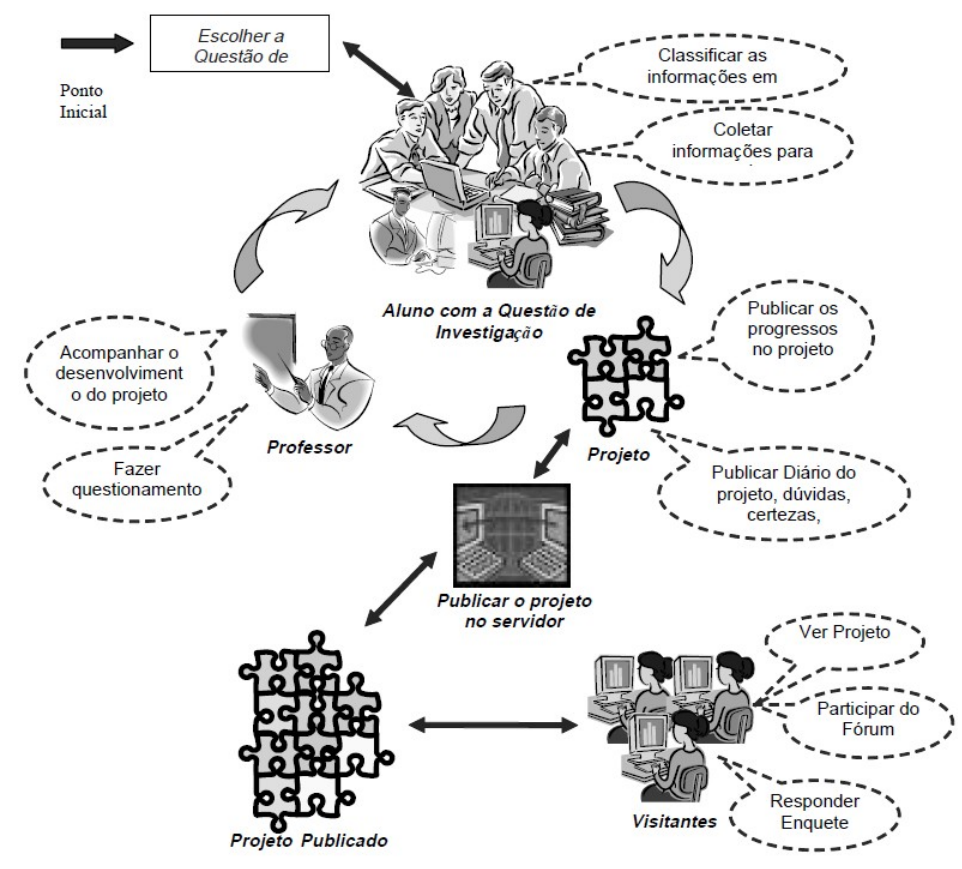

Figura 1: Processo de desenvolvimento de Projetos de Aprendizagem. (Carvalho et al., 2005).

Os atores envolvidos (figura 1) durante o processo de implementação do projeto de aprendizagem nos MV são: o mediador, o estudante e o colaborador. O estudante possui com o papel de protagonista, é o construtor que tem a informação sobre os melhores caminhos para desenvolver sua construção, além disso, é autônomo e original no seu processo de aprendizagem. O mediador é o especialista, o articulador, o orientador e parceiro desta empreitada. O mediador não é aquele que apresenta as respostas, mas aquele que apresenta os meios para que os aprendizes construam suas 
próprias respostas. O grupo de colaboradores (visitantes) tem um papel importante nesse processo e pode apoiar os aprendizes e mediadores com novas opiniões sobre o projeto e seus resultados, também pode participar como um coautor das pesquisas que forem sendo realizadas.

Observamos que, a partir da proposta dos PAs, a organização dos domínios tem como foco o aprendizado entre professores e os estudantes. O estudante é desafiado a questionar, argumentar e construir.

É dada também a ele a capacidade de formular, equacionar e virtualizar. É permitido propor questões que possuem significações. Estas questões de significações emergem de sua história de vida, de seus interesses, de seus valores, de suas necessidades e de suas condições pessoais. Ao apoiar a construção de Projetos de Aprendizagem, por intermédio de mundos virtuais, alguns requisitos básicos devem ser observados, como cooperação entre os indivíduos, construção e reconstrução, e equilíbrios e desequilíbrios.

Os Projetos de Aprendizagem usam e produzem informações que necessitam ser interligadas em forma de rede para que os desenvolvedores possam rever lições aprendidas, revisar decisões tomadas, reaproveitar informações e gerar documentos de variadas linguagens: textos, imagens, vídeos, tabelas, mapas de conceitos, gráficos, animações, jogos, apresentações, etc. (Fagundes et al., 2006). Neste sentido, um MV pode proporcionar a cooperação entre seus usuários, devidamente representados por seus avatares, a autoria de novos elementos e estratégias para solução de problemas e, ainda, deve possibilitar a manipulação dos componentes integrantes do ambiente.

\section{Os Mundos Virtuais Avaliados}

Para realizar a avaliação dos MVs, mapeamos etapas para essa metodologia. Primeiro, propõe-se, aqui, a título de prova de conceito, utilizar os MVs para a realização de projetos de aprendizagem e, depois, realizamos uma avaliação.

\subsection{Escolha dos Mundos Virtuais}

A escolha dos MVs foi por uma seleção realizada seguindo alguns critérios: popularidade, estabilidade, disponibilidade e o tipo de licença. A popularidade foi caracterizada pela constatação de citações em artigos da área ao longo de várias leituras realizadas. A estabilidade observa os impactos gerados na mudança das versões anteriores, o que mostra maturidade do software. Já a disponibilidade é vista quando o mundo está em um servidor que fornece o serviço continuo. Por último, e de suma importância, o tipo de licença deve ser de uso gratuito.

Os MVs escolhidos neste artigo para a realização da avaliação foram: SecondLife (Second Life, 2012), Active Worlds (Active Worlds, 2012) e o Habbo Hotel (Habbo, 2012).

\subsubsection{Second Life}

O Second Life (SecondLife, 2012) oferece aos usuários a experiência de participar com avatares, que podem ser personalizados de formas variadas (roupa, estilo, sexo, entre outras características). É uma experiência social, com usuários capazes de ver e se comunicar uns com os outros, tanto em um mundo público quanto privado, por meio das mídias, incluindo mensagens instantâneas e um sistema de comunicação de voz.

O Second Life (SL) oferece aos usuários uma experiência de participar em um MV 
como avatares animados, que podem ser personalizados de diversas maneiras, podendo ser objetos, animais e pessoas. Suas habilidades são caminhar, voar, teletransportar, também têm a capacidade de se comunicar por meio de mídia, incluindo mensagens instantâneas e sistema de comunicação de áudio (Lyons, 2008).

No SL, os minimundos são chamados de ilhas. Algumas inconveniências são encontradas nesse mundo durante uma visita, por exemplo, para o uso nas escolas, ao passo que teremos ao realizar uma dinâmica com os alunos: nem todas as ilhas são públicas, não existe um código de ética nas ilhas, existe uma grande reclamação da sua lentidão, os computadores nas escolas não conseguem executar de maneira satisfatória, fugindo da realidade social que foi mencionada (Santos, 2011).

\subsubsection{Active Worlds}

O Active Worlds (AW) (ActiveWorlds, 2012), é visto não como um ambiente virtual multiusuário, mas como uma plataforma de criação de conteúdos em 3D, já que é possível realizar essas construções, em que os usuários são representados por um avatar e é possível que conversem, explorem e construam coisas nos seus próprios mundos virtuais. A primeira versão foi denominada AlphaWorld, que depois foi chamada de Active Worlds e lançada, oficialmente, em 1995. Nesse mundo, existem 2 dois tipos de usuário: turista e cidadão.

Para o acesso como um turista, não é cobrado nada, porém há limitação em acesso, e em algumas áreas e ações. Já o acesso como cidadão é amplo, porém é necessário pagar uma taxa mensal. Há inclusive um calendário de eventos que apresenta festas e encontros, bastando selecionar o dia. No cliente, são apresentados todos os mundos disponíveis e a quantidade de usuários presentes em cada um deles. Um dos usos e possibilidades do AW é que possui um ambiente voltado para mundos totalmente com fins educacionais. Por meio dele, educadores e estudantes podem trabalhar conceitos e teorias de aprendizagem de uma forma diferente da tradicional, construindo seus próprios mundos e objetos (Dickey, 2003).

\subsubsection{Habbo Hotel}

Habbo Hotel é um mundo virtual que proporciona aos jovens poder se sentir participante de um ambiente diferente do seu cotidiano, é considerado o maior jogo social destinado ao público jovem no mundo. Possui 11 (onze) versões de idiomas diferentes e possui 226 milhões de usuários registrados em mais de 150 países. O Habbo Hotel recebe seus hóspedes de maneira gratuita e permite a eles a criação de quartos que podem ser decorados com mobílias virtuais, compradas com as Habbo Moedas, o dinheiro virtual válido no jogo (Lehdonvirta et al., 2009).

Por ser um local para encontrar e fazer novas amizades, este jogo trata-se de um ambiente no qual o usuário cria gratuitamente uma personagem virtual que interage com as personagens eletrônicas de outros internautas, as conversas podem ser realizadas via chat. Além do contato com outros jogadores em espaços públicos, o usuário pode criar seus próprios quartos que podem ser decorados com mobílias virtuais, que, uma vez vazios, convidam o jogador a decorá-los da maneira que preferir, desde que se oriente pelo catálogo de mobílias do hotel (Habbo, 2012).

\subsection{Elicitação dos Requisitos}

Para avaliar os mundos virtuais escolhidos, foi utilizado como base Projetos de Aprendizagem, conforme Fagundes et al. (2006). Para isto, foram definidos alguns 
critérios para que fosse possível a realização desta avaliação. Comunicação entre os participantes é a capacidade de um mundo virtual permitir que ocorra comunicação via vídeo, texto ou voz com os participantes. Suporte para formação de grupos é a possibilidade de um mundo virtual permitir a formação de equipes/grupos para que possam cooperar entre eles. Visibilidade dos participantes é um requisito que possibilita visualizar todos os indivíduos que estão participando no mundo virtual/aventura. Definir prazos é a capacidade de um mundo virtual definir prazo para que uma atividade possa ser finalizada/consultada ou a duração de uma aventura/atividade. Criação de espaço é a característica para que os indivíduos tenham a flexibilidade em criar seus espaços dentro do mundo. Definir Desafios é o requisito que novos desafios/questionamentos sejam propostos durante a aventura no mundo virtual. Criação de propriedade intelectual é a flexibilidade que existe no mundo virtual para que o indivíduo possa ser capaz de criar objetos, textos, som, vídeos e imagens durante a aventura, e estes possam ser utilizados durante a aventura. Criação de enredo é um requisito que estabelece a possibilidade da criação de uma narrativa/história dentro do mundo virtual. Suporte para anotações de certezas/dúvidas é o requisito que permite ao estudante realizar anotações em um editor para que fiquem armazenadas suas certezas e dúvidas durante a aventura. Determinação de papéis é a capacidade do mundo virtual realizar, de forma dinâmica, a atribuição de perfis aos indivíduos. Convite para novos usuários é o requisito que permite ao mundo virtual submeter um convite (ou um aviso) para que os indivíduos realizem o cadastro no ambiente. Compartilhamento de recursos é a possibilidade do indivíduo compartilhar objetos e recursos multimidiáticos que estão disponíveis no mundo virtual. Monitoramento é a possibilidade do mediador acompanhar as atividades realizadas pelo indivíduo. Suporte para colaboradores externos é um requisito que possibilita a colaboradores que não estão dentro da aventura, de alguma forma, colaborar opinando e participando de possíveis pesquisas. Suporte a Questionamentos é o requisito em que o mediador poderá questionar com perguntas/sugestões durante a vivência no mundo virtual. Cooperação entre os indivíduos é a possibilidade dos indivíduos ajudarem uns aos outros com tarefas e novas descobertas.

\section{Avaliação e Resultados}

Após o levantamento dos requisitos para avaliação do PA, realizamos a experimentação da implementação nos mundos virtuais selecionados. A tabela 1 exibe uma compilação dos resultados extraídos do experimento que foi realizado. Foi definida uma nota qualitativa a ser atribuída a cada ambiente, segundo a conformidade com cada requisito, que foram: [3] - atende plenamente a descrição do requisito; [2] - atende parcialmente e requer pequenas adaptações; [1] - atende parcialmente e requer muitas adaptações; e [0]

- não há recursos que suportem o requisito. 


\begin{tabular}{|c|c|c|c|}
\hline $\begin{array}{c}\text { Requisitos/Mundos } \\
\text { Virtuais }\end{array}$ & $\begin{array}{l}\text { Second } \\
\text { Life }\end{array}$ & $\begin{array}{l}\text { Active } \\
\text { Worlds }\end{array}$ & $\begin{array}{c}\text { Habbo } \\
\text { Hotel }\end{array}$ \\
\hline $\begin{array}{l}\text { Comunica ção entre os } \\
\text { participantes }\end{array}$ & 3 & 3 & 1 \\
\hline $\begin{array}{l}\text { Suporte para formação de } \\
\text { grupos }\end{array}$ & 2 & 2 & 0 \\
\hline $\begin{array}{l}\text { Visibilidade dos } \\
\text { participantes }\end{array}$ & 2 & 1 & 1 \\
\hline Definir prazos & 1 & 1 & 0 \\
\hline Criação de espaços & 1 & 2 & 0 \\
\hline Definir desafios & 1 & 1 & 1 \\
\hline $\begin{array}{l}\text { Criação de propriedade } \\
\text { intelectual }\end{array}$ & 2 & 2 & 1 \\
\hline Criação de enrendo & 2 & 2 & 0 \\
\hline $\begin{array}{l}\text { Suporte para anotações de } \\
\text { certezas/dúvidas }\end{array}$ & 1 & 1 & 0 \\
\hline Determinação de papé is & 2 & 2 & 1 \\
\hline $\begin{array}{l}\text { Nivel de satisfação dos } \\
\text { Individuos }\end{array}$ & 2 & 2 & 2 \\
\hline $\begin{array}{l}\text { Compartilham ento de } \\
\text { recursos }\end{array}$ & 2 & 2 & 2 \\
\hline Monitoramento & 1 & 1 & 1 \\
\hline $\begin{array}{l}\text { Suporte para } \\
\text { colaboradores extemos }\end{array}$ & 2 & 2 & 0 \\
\hline $\begin{array}{l}\text { Suporte a } \\
\text { questionamentos }\end{array}$ & 2 & 2 & 1 \\
\hline $\begin{array}{l}\text { Cooperação entre os } \\
\text { Individuos }\end{array}$ & 3 & 3 & 3 \\
\hline $\begin{array}{l}\text { Suporte para avaliação pré } \\
\text { e pós }\end{array}$ & 1 & 1 & 1 \\
\hline $\begin{array}{l}\text { Aplicação em situações } \\
\text { concretas ao mundo real }\end{array}$ & 3 & 3 & 3 \\
\hline
\end{tabular}

Tabela 1: Tabela de avaliação dos requisitos do Mundo Virtual

Partindo da avaliação realizada os mundos virtuais avaliados possuem algumas limitações e apresentam grandes possibilidades para que possam suportar projetos de aprendizagem.

Os MVs são poucos flexíveis para apoiar tais práticas pedagógicas, que requerem monitorações e coordenação, e estas são características que não foram projetadas quando desenvolvidos tais mundos virtuais.

\section{Conclusão}

Portanto, Mundos Virtuais possuem um suporte telemático capaz de satisfazer a aprendizagem utilizando-os para a metodologia de ensino. Porém, constatamos que ainda requerem adaptações com finalidade de melhor se adequar aos requisitos do mundo virtual quando realizados trabalhos com suporte a projetos de aprendizagem.

Neste artigo, evidenciamos a possibilidade de novos caminhos para a construção de softwares educacionais que se constituem em uma alternativa para o uso de tecnologias como suporte à promoção da aprendizagem. Conforme a avaliação realizada dos MVs, percebemos que são necessários ambientes com ferramentas pedagógicas possibilitando um melhor acompanhamento dos alunos durante a vivência em mundos virtuais. 


\section{Referências Bibliográficas}

Activeworlds Inc. (2012). Active Worlds Tour. Disponível em: http://www.activeworlds.com/. Acessado em 01/10/2012.

Bell, B. M. W. (2008). Toward a Definition of "Virtual Worlds" Toward a Definition of "Virtual Worlds". Jornal of Virtual Worlds Research. ISSN 1941-8477. Volume 1.

Carvalho, M.J. S., Nevado, R.A., Menezes, C.S. (2005). Arquiteturas Pedagógicas para Educação a Distância: Concepções e Suporte Telemático, Simpósio de Informática na Educação, Juiz de Fora - MG.

Daden, L. (2010). Virtual Worlds for Education and Training. Disponível em < http://www.daden.co.uk/downloads/Virtual\%20Worlds\%20for\%20Training\%20and \%20Education\%2001d2.pdf> Acessado em 11/05/2014.

Dickey, M.D. (2003). 3D Virtual Worlds: An Emerging technology for traditional and Distance Learning. Proceedings Ohio Learning Network; The Convergence of Learning and Technology - Windows on the Future. Ohio, USA.

Fagundes, L., Nevado, R., Basso, M., Bitencourt, J., Menezes, C., Monteiro, V.C.P.C. (2006) “ Projetos de Aprendizagem - Uma experiência mediada por ambientes Telemáticos”. Revista Brasileira de Informática na Educação.

Fagundes, L. Sato, L. S.; Maçada, D. L. (1999) “Aprendizes do Futuro: as Inovações Começaram", Revista Coleção Informática para a Mudança na Educação. Brasília: MEC/SEED/PROINFO.

Gee, J. P. (2003) "What Video Games Have to Teach Us About Learning and Literacy", ACM Computers in Entertainment, Vol. 1, No. 1, BOOK 1.

Gee, J. P. (2007). Good Video Games + Good Learning. Collected essays on videogames, learning and literacy. Peter Lang Publishing. New York.

Habbo Hotel (2012). Disponível em: <http://www.habbo.com//>. Acessado em 01/10/2012.

Lehdonvirta, V.; Wilska, T.; Johnson, M. (2009), Virtual Consumerism: Case Habbo Hotel. Information, Communication \& Society, v. 12 n. 7, Helsinki Institute for Information Technology, Finland.

Lyons, K. Towards a Theoretically-Grounded Framework for Evaluating Immersive Business Models and Applications: Analysis of Ventures in Second Life. University of Toronto. Jornal of Virtual Worlds Research. Julho, 2008.

Mattar, J. Games em educação: como os nativos digitais aprendem. 1 ed. Sã Paulo: Pearson Prentice Hall, 2009.

Pessoa, J. M.; Menezes, C. S. (2003) Framework Baseado em Padrões Abertos para Construção de Ambientes CSCW/CSCL na Web, in Proceedings of the First Latin AmericanWeb Congress -La-Web. 0-7695-2058-8/03. IEEE. 2003.

Prensky, M. (2001). "Digital natives, digital immigrants. On the Horizon. University Press. Vol. 9. N 5.

Prensky, M. (2007). "Students as Designers and Creators of Educational Computer Games". 
Santos, O. L., Rafalski, J. P., Silva, H. F. A., Cury, D., Menezes, C. S. (2011) "Mundos Virtuais para Construção de Arquiteturas Pedagógicas", Workshop WAPSEDI Anais do XXII SBIE - XVII WIE.

SecondLife (2012). Disponível em: http://secondlife.com/. Acessado em 01/10/2012.

Schlemmer, E.; Backes, L. (2007). O Aprender e o Ensinar na Formação do Educador em Mundos Virtuais. In: Educere et Educare Revista de Educação.- Cascável. Paraná, RS. Anais disponível em versão impressa e eletrônica. Unioeste Universidade Estadual do Oeste do Paraná. 